\title{
Effect of Alcohol on Central Nervous System and HIV Infection: A Study on Dhankuts Living in District Bahraich of Uttar Pradesh, India
}

\author{
Alok Chantia and Preeti Misra \\ Department of Anthropology, Sri Jai Narain Post Graduate College, Lucknow, \\ Uttar Pradesh, India \\ E-mail: alokchantia@gmail.com \\ Department of Human Rights, School for Legal Studies, \\ Babasaheb Bhimrao Ambedkar University, Lucknow, Uttar Pradesh, India \\ E-mail: misra9us@gmail.com
}

KEYWORDS HIV. Alcohol. Sexuality. Central Nervous System. Dhankut. Culture

\begin{abstract}
Human Immunodeficiency Virus (HIV) is endangering humanity. Many people at risk of or infected with the HIV are heavy drinkers. Both HIV infection and heavy alcohol use adversely affect the immune system and central nervous system (CNS) function. However, little research has addressed the effects of heavy alcohol use on the severity and progression of HIV disease, including the development of HIV associated CNS disease. Animal and in-vitro studies suggest that alcohol impairs various aspects of the immune system and increases the susceptibility to HIV infection, but may not accelerate progression of HIV disease. However, heavy alcohol use may interfere with the patient's adherence to antiretroviral treatment regimens. Neuropathological and neuropsychological studies have indicated that certain brain areas are affected by both HIV infection and chronic alcohol abuse. Magnetic resonance spectroscopy studies of both HIV positive and HIV negative people who were either heavy or light drinkers found that chronic alcohol abuse exacerbates some metabolic injury in the brains of HIV infected people, although this effect may be less pronounced in patients receiving effective antiretroviral therapy. Present study has been done on an endogamous group, Dhankut, living in Dhankuttypura of Bahraich district of Uttar Pradesh, India. The object of present paper is to see how alcohol impairs various aspects of the immune system and increases the susceptibility to HIV infection amongst this group. The paper also analyses the effects of heavy alcohol use on the severity and progression of HIV infection, including the development of HIV associated CNS disease with the help of various studies done on the subject.
\end{abstract}

\section{INTRODUCTION}

Infection with the human immunodeficiency virus (HIV) and the resulting acquired immunodeficiency syndrome (AIDS) epidemic is one of the major public health problems in the world. One common cause of illness and death associated with HIV infection is central nervous system (CNS) disease. The most severe manifestation of CNS disease is HIV-associated dementia complex (HADC), a neurological syndrome characterized by disordered mental functions (that is, cognition), motor functions, and behavior. Many members of populations at high risk for HIV infection (for example, intravenous drug users and homosexuals) also are heavy drinkers, and alcohol has its own adverse effects on CNS function. Nevertheless, almost no research has directly addressed the effects of heavy alcohol use on the severity of HIV disease and its progression to AIDS.

This paper summarizes information regarding the prevalence of alcohol abuse among HIVinfected people as well as current knowledge regarding the separate and combined effects of HIV infection and alcohol use on the brain and the immune system. The article also reviews the relationship among alcohol use and sexual risks, adherence to HIV treatment regimens, and HIV disease progression. It discusses the CNS effects of HIV infection/AIDS and alcohol abuse, focusing on pathological and psychological observations as well.

\section{Prevalence of Chronic Alcohol Use among the HIV-Infected Population}

By the end of the year 2000, approximately 920,000 people were living with HIV infection/ AIDS, and approximately one-half of these patients had been infected with HIV for more than 10 years. Most HIV-infected people are between ages 18 to 44 . Within the general po-pulation of this age range, 17 percent of men and 10 percent of women are afflicted at some point over their lifespan by an alcohol use disorder (AUD) - that is, alcohol abuse or dependence. Among alcohol-abusing patients, the HIV infection rate 
is significantly higher than for the general population-that is, about 5 to 10 percent (depending on risk factors such as intravenous drug use, homosexuality, and geographic location). (For a more extensive review of alcohol use in HIVinfected patients see, Petry 1999.)

Similarly, AUDs are much more common among HIV-infected people than among the general population. For example, 29 to 60 percent of HIV-infected patients develop an AUD at some point during their lives, a rate that is approximately three times as high as that of the general population. In fact, this prevalence rate is one of the highest rates of drug abuse found among any population group. Moreover, the prevalence of a current AUD among HIV-infected patients is nearly 12 percent, about twice the rate of the general population (Petry 1999). AUDs may affect HIV-associated CNS disease in two ways. First, heavy chronic drinking may hasten the progression of the overall HIV disease process through biological (for example, immunological) effects. Second, AUDs may interfere with treatment seeking, treatment adherence, and treatment effectiveness among HIVinfected people.

\section{Biological and Immunological Effects of HIV Infection and Chronic Alcohol Use}

\section{Targets of HIV Infection in the Immune System and CNS}

The primary target of HIV in the body is a type of immune cell called the CD4+, or T-helper cell, 3 which plays a pivotal role in the body's defense against disease-causing micro-organisms (that is, pathogens). HIV-infected CD4+ cells produce new virus and eventually die. As a result, the infected person's levels of CD4+ cells in the blood slowly decline, leaving him or her increasingly vulnerable to infections by various pathogens that normally are controlled easily by the immune system (that is, opportunistic infections) and to unusual cancers.

CD4+ cells are not found in the brain, however, and HIV therefore must infect other cells there to cause CNS disease. The primary cell types in the CNS are nerve cells (that is, neurons) and glial cells, which include the following three major subtypes:

- Astrocytes, which provide structural and functional support for neurons and which are important for the regulation of ion concentrations

- Oligodendrocytes, which extend projections that form a protective sheath (that is, myelin sheath) around the extensions (that is, axons) of neurons

- Microglia, which can change into a type of immune cell called phagocyte or macrophage that seeks out and destroys foreign microorganisms and viruses.

HIV does not infect neurons, but it does infect microglia and, to a lesser extent, astrocytes. Once HIV has entered the brain, these "activated" microglia and multiplying astrocytes produce increasing amounts of substances that when present at excessive levels are toxic to neurons (that is, neurotoxins). The production of neurotoxins is stimulated by compounds called cytokines that act as chemical messengers among immune cells as well as between immune and nonimmune cells. The same cytokines also further enhance the multiplication of astrocytes and microglia (that is, gliosis) as well as the production of new HIV particles.

Neurotoxins can damage or even kill neurons, and this process is thought to contribute to the neuropsychological and cognitive deterioration that occurs during the natural course of HIV disease. The most serious consequence of this deterioration is HADC, which is found in approximately 15 percent of HIV-infected people, mostly in those patients whose immune systems have already been severely damaged by HIV (that is, patients who are severely immune suppressed).

\section{Effects of Alcohol on the Immune System}

Chronic heavy alcohol use is associated with increased illness and even death related to infectious diseases, although alcohol's specific effect on the immune system in these relationships is controversial. Limited studies conducted in intact animals (that is, in vivo) and in cultured tissues and cells (that is, in vitro) suggest that alcohol can interfere with the normal functions of various components of the immune system (for example, natural killer cells and Tcells), thereby impairing the body's response to pathogens (for a review, see Szabo 1999). However, little is known about the effects that acute and chronic alcohol consumption have on the function of the peripheral (that is, not pertain- 
ing to the CNS) immune system in humans, and no information exists about alcohol's effects on the immune function of the CNS (that is, on the microglia). Animal and human studies suggest that chronic alcohol consumption adversely affects the peripheral immune system, probably by inducing the production of cytokines. If a similar mechanism exists in the CNS, alcoholinduced cytokine release could increase the amount and activity of neurotoxins and, subsequently, lead to the death of neurons. This hypothesis of alcohol-induced neuron damage is consistent with the findings of neuro-pathological and imaging studies of chronic alcohol abusers.

\section{Effects of Combined HIV Infection and Alcohol Use on the Immune System}

The previous two sections suggest that both HIV infection and chronic heavy drinking have similar and profound effects on the immune system (for extensive reviews, including immunological and behavioral concepts, see Dingle and Oei 1997; Petry 1999; Tyor and Middaugh 1999). Therefore, researchers have postulated that chronic alcohol use by HIV-positive people may exacerbate the immunological abnormalities that are associated with HIV infection. Researchers have begun to analyze the effects of alcohol in HIV-infected subjects using rodent models of HIV infection, in which mice are infected with a virus that is similar to HIV and can develop a condition comparable to AIDS. These studies suggest that both acute and chronic alcohol consumption reduce the animals' peripheral immune functions, possibly by altering the release of certain cytokines (for reviews, see Dingle and Oei 1997; Tyor and Middaugh 1999). Furthermore, the animals progress more rapidly from the initial viral infection to mousetype AIDS or death.

Researchers also have conducted studies using immune cells (that is, lymphocytes) -including CD4+ cells - that were isolated from healthy, non-HIV-infected human subjects both before and after the subjects consumed 3 to 9 alcoholic drinks over 2 days. The studies suggested that alcohol consumption damaged the peripheral immune system, because when the isolated cells were infected with HIV in a test tube, the virus multiplied faster in cells taken after alcohol consumption. Furthermore, cells isolated after alcohol consumption exhibited reduced CD4+ functions compared with cells isolated before alcohol consumption. These findings suggest that alcohol increases the susceptibility of human cells to HIV infection. Alcohol may exert these effects either directly, by acting on CD4+ cells, or indirectly, by altering the production of cytokines. Some of these effects may be reversible, however, because studies on alcoholic HIV-infected patients undergoing alcoholism treatment found that the patients' levels of CD4+ cells improved after detoxification (Pol et al. 1996).

Although it appears likely that alcohol impairs the activity of the peripheral immune system and increases the susceptibility to HIV infection, the effects of alcohol on human immune function in the CNS and on the susceptibility of brain cells to HIV infection are ambiguous. Similarly, the impact of alcohol use on the progression of HIV disease to AIDS remains unclear. Several immunological studies followed large numbers of alcohol-consuming homosexual men over time but found no evidence that alcohol accelerated disease progression to AIDS ( Kaslow et al. 1989; Penkower et al. 1995). These studies have methodological weaknesses, however. For example, one study followed the participants only over a period of 18 months, which may not have been long enough, considering that it usually takes approximately 3 to 10 years for an HIV-infected person to develop AIDS. Another study only examined the effects of consumption of upto approximately 70 alcohol-containing drinks per month, or a little more than 2 drinks per day, which generally is considered moderate drinking; the study did not assess the consequences of heavier drinking. A more recent report suggests, however, that even heavy drinking (that is, more than 150 drinks per month for an unspecified duration) does not significantly alter the effectiveness of antiretroviral treatment in controlling the virus levels in HIV-infected patients (Fabris et al. 2000). This finding also implies that alcohol consumption does not accelerate the progression of HIV disease.

In summary, animal and in-vitro studies have indicated that chronic alcohol use can have deleterious effects on the immune system. In addition, chronic alcohol use may influence a person's susceptibility to infections by modifying the nutritional status and promoting the 
generation of highly reactive molecules that can damage the cells (that is, oxidative stress) (see Wang and Watson 1995). Thus, it appears plausible that heavy drinking would increase a person's susceptibility to infection by HIV and accelerate disease progression to AIDS. To date, however, no strong immunological evidence links chronic alcohol consumption at any level to an accelerated course of HIV disease. The paucity of existing immunological data demonstrates a great need for well-controlled, preferably long-term studies to document the combined effects of HIV infection and heavy alcohol consumption on the progression of HIV disease in this era of promising antiretroviral therapy.

\section{Effects of HIV Infection and Alcohol Abuse on the CNS}

The development of new antiretroviral medications has greatly enhanced treatment effectiveness in controlling the spread of HIV in the body. These medications cannot effectively enter the CNS, however, to eradicate the virus that has infected brain cells. Accordingly, factors that influence CNS disease, such as alcohol abuse, may become even more important in the clinical management of HIV disease and of the HIV epidemic. The separate effects of HIV infection and chronic alcohol abuse on brain structure and function and on biologically relevant molecules that are associated with neuron loss and gliosis are well established. However, almost no research has addressed directly the effect of heavy alcohol use on the severity and progression of HIV-associated CNS disease. This section reviews the main CNS effects of both HIV infection and alcohol abuse by summarizing the pathological and psychological evidence for brain damage associated with both conditions. This discussion also describes structural and some functional correlates of such damage, which can help to understand the cognitive changes occurring in alcohol-abusing HIV-infected patients.

\section{Neuropathology and Neuropsychology of HIV-Infected People}

Neuro-pathological studies conducted during the 1990s found that approximately 75 percent of the AIDS patients studied exhibited some kind of CNS damage. Furthermore, a similar proportion of AIDS patients exhibited neurological signs or symptoms before their death, such as memory loss, decreased concentration and attention, and problems with movement or balance (Price 1999). Neuro-pathological studies also revealed virus-associated abnormalities in various brain areas, including the following (Cummings 1986; Price et al. 1988)

- The Basal Ganglia. These are groups of nerve cells located deep inside the brain that help control motor and cognitive activity. The basal ganglia region carries the heaviest HIV load of all brain structures and shows effects of gliosis and inflammation early in the course of the disease. Neuron loss in this region, although it is not a common finding, has been reported in some more recent studies (Masliah et al. 1996).

- White Matter Around the Fluid-filled Spaces (that is, ventricles) in the Brain. White matter consists primarily of glial cells and axons surrounded by myelin sheaths. In HIV-infected patients, this white matter is infiltrated by various immune cells (that is, macrophages and lymphocytes). Patients with advanced stages of HIV disease often exhibit abnormal proliferation of glial cells and changes in the myelin sheaths.

- The Cortex. The cortex is the brain's outer layer, which is responsible for higher cognitive functions. In advanced stages of AIDS, cortical changes have been reported, including loss of large motor neurons, decreased density of the neuron structures that allow nerve signal transmission among neurons (that is, synapses), and damaged neuronal extensions (that is, dendrites) that normally receive incoming nerve signals. Consistent with these observed abnormalities, HADC patients in neuropsychological studies exhibit a constellation of deficits associated with dementia resulting from damage to brain regions other than the cortex (that is, subcortical dementia), including deficits in concentration and attention, psychomotor slowing, and mild-to-moderate impairments of memory and abstraction ability (Cummings 1986). Similar impairments are sometimes observed in alcoholic patients. Even though many $\mathrm{HIV}$-infected people are heavy drinkers, a study 
comparing the neuropsychological functions of HIV-positive and HIV-negative participants suggested that alcohol use did not account for differences in the test scores between the two groups (Bornstein et al. 1993). HAART has been shown to improve neuropsychological test performance of HIV-infected people in some but not all cognitive domains (Tozzi et al. 1999).

\section{Brain Structure and Metabolites in Chronic Alcohol Abusers}

Consistent with the neuropathological studies, imaging studies using computed tomography (CT) scans demonstrated atrophy in various brain regions of alcoholics as indicated by shrinkage of the cerebellum and widening of the ventricles and fluid-filled gaps (that is, sulci) in the brain's cortex, particularly in the frontal lobe (for reviews see Rosenbloom et al. 1995; Sullivan 2000). MRI studies also demonstrated volume loss in brain regions that show neuron loss in pathological studies, primarily in cortical gray matter regions of the frontal lobes, but also in gray matter of the parietal and temporal lobes. This gray matter loss often is accompanied by increases in fluid volume in the same regions. In addition, MRI studies of chronic alcoholics have demonstrated white matter loss in the frontal-parietal brain and corpus callosum, as well as volume loss of sub-cortical structures (for example, the thalamus, hypothalamus, and caudate) and the cerebellum. As did neuropa-thological studies, neuro-imaging studies found no decrease in the size of the hippocampus, despite the memory and learning deficits observed in alcoholics. Researchers have demonstrated some relationships between volume deficits in some brain areas and measures of neuropsychological impairment; however, these relationships have not been consistent.

An alcoholic's age influences how severe alcohol-related changes in brain structure and function are, with more severe and persistent alcohol-related changes evident in the brains of older alcoholics (Sullivan 2000). Age also appears to affect the brain's ability to recover from alcohol-related damage. When alcoholics stop drinking, some of the gray and white matter loss and cognitive impairment caused by chronic drinking is reversible. Studies found that the recovery of brain structure and function is greater and more rapid in younger abstinent alcoholics than in older abstinent alcoholics (see, Sullivan 2000). The significance of the recovery of brain structures for the recovery of brain functions is under active investigation.

Despite their potential usefulness, MRS studies on the effects of chronic alcohol abuse on the brains of humans or animals are scarce (see, Sullivan 2000). Studies of abstinent alcoholics generally suggest lower NAA levels in white matter regions of the frontal and parietal lobes as well as in the cerebellum and thalamus, consistent with neuronal and/or axonal damage in those regions that was not reversible by short-term abstinence. These observations support those of the neuro-pathological and neuro-psychological studies described in the previous section. Patients with alcoholic dementia also reveal evidence of neuronal damage in the basal ganglia and occipital lobe. One MRS study compared certain brain metabolites in the thalamus and frontal cortical regions between alcoholics who had been abstinent for 1 month and alcoholics who had been abstinent for 6 years (see, Sullivan 2000). The study found elevated mIno levels after short-term abstinence but not after long-term abstinence, suggesting that some alcohol-induced changes are reversible with prolonged abstinence.

\section{Comparison of the Brain Regions Affected by HIV Infection and Chronic Alcohol Abuse}

Taken together, the pathological, neuro-psychological, structural, and metabolic evidence suggests that chronic alcohol abuse predominantly affects the frontal lobe whereas HIV - at least early in the course of the disease-primarily affects white matter deep within the brain as well as sub-cortical brain structures (see, Table 1). Cortical brain regions become involved only during advanced stages of HIV disease, which are associated with cognitive and some clinical impairment. Furthermore, this cortical damage may be delayed by HAART therapy.

Based on these findings, the brain regions expected to be primarily affected in alcoholabusing HIV-infected subjects include central white matter, sub-cortical brain structures, and the frontal cortex. These regions are the focus of current structural and metabolic MR studies of the combined effects of alcohol abuse and HIV infection on the brain. 


\section{CNS Effects of Combined HIV Infection and Chronic Alcohol Abuse}

The findings reviewed so far strongly suggest that chronic alcohol abuse can adversely affect the natural course of HIV disease. To prove, however, that chronic alcohol abuse exacerbates the CNS effects of HIV infection, researchers must compare alcohol-abusing and non-alcohol-abusing HIV-infected patients on a variety of CNS measures. This section summarizes studies analyzing the interactive effects of HIV infection and chronic alcohol abuse on the brains of patients not treated with HAART. Using MRS of the brain, both HIV-positive and HIV-negative subjects were examined who were either heavy drinkers (that is, consumed a mean of 228 drinks per month for at least 3 years prior to study) or light drinkers (that is, consumed a mean of 14 drinks per month over their lifetime). The light-drinking and heavy-drinking HIV-positive groups were matched for the degree to which their immune systems were already impaired by the HIV infection, and both groups exhibited mild-to-moderate neuropsychological impairment. The study assessed the levels of two molecules called PCr and ATP, which reflect the cell's energy metabolism, as well as of a group of compounds called PDE, which represent breakdown products of molecules found in the cell membranes.

The study found that chronic alcohol consumption was associated with lower concentrations of PDE, PCr, and ATP in white matter of the region surrounding the ventricles (that is, the centrum semiovale). Similarly, patients with advanced HIV disease or full-blown AIDS had lower levels of PDE, PCr, and ATP in the white matter compared with both HIV-negative subjects and clinically asymptomatic HIV-positive subjects. The metabolic effects of advanced HIV infection and alcohol abuse were cumulative, because HIV-positive heavy drinkers clearly showed the greatest metabolic deficits in white matter. PDE levels also were significantly lower in sub-cortical gray matter, which is associated with learning and memory functions, of heavy drinkers with advanced HIV disease compared with HIV-negative light drinkers. These findings are consistent with the neuro-pathological and neuro-psychological observations demonstrating sub-cortical damage.

Taken together, these findings suggest that both alcohol abuse and HIV infection alter the brain's energy metabolism and the metabolism of cell membranes and that these alterations are associated with the presence of clinical HIV symptoms. More importantly, the study found that chronic alcohol abuse augmented the adverse effects of HIV on biologically important white matter molecules.

MRS study has been conducted assessing different molecules, including NAA, in a similar patient population. That study found metabolic changes in the regions of the brain stema region at the base of the brain that performs motor, sensory, and reflex functions - of active heavy drinkers. These changes were consistent with pathological evidence for the loss of certain neurons and for gliosis found throughout the brain stem of chronic alcoholics. These changes were particularly pronounced in HIVpositive heavy drinkers, suggesting that HIV infection may have an additional adverse effect on brain stem metabolites.

These studies, which were performed before the introduction of HAART therapy, suggest that chronic alcohol abuse exacerbates some metabolic injury in the HIV-infected brain. Electrophysiological studies, which measure certain aspects of the brain's electrical activity, have provided additional evidence for an additive effect of heavy drinking on brain damage in HIV-infected subjects. One study assessed an electric signal measurable in the frontal lobe that is thought to reflect mental processes requiring attention and memory updating (Fein et al. 1998). This signal is delayed in HIV-infected subjects, but the delays occurred earlier in the course of HIV disease in alcohol-abusing than in light-drinking or abstinent HIV-infected subjects. Researchers are currently investigating the cognitive and behavioral significance of these brain changes.

The effect of modern antiretroviral treatment (that is, HAART therapy) on the specific brain alterations observed in alcohol-abusing HIVpositive patients has not yet been investigated thoroughly. Very preliminary data from MRI and MRS studies on light- and heavy-drinking HIV-negative and HIV-positive subjects (with most of the HIV-positive subjects on HAART therapy) detected some of the brain injury commonly associated with HIV infection and with alcohol abuse. The studies found little evidence, however, that chronic alcohol abuse 
exacerbated the CNS effects of HIV infection in these treated patients.

Table 1: Brain regions affected by HIV infection and chronic alcohol abuse based on pathological, psychological, structural, and metabolic analyses

\begin{tabular}{lll}
\hline Brain region & $\begin{array}{l}\text { Affected by } \\
\text { HIVinfection }\end{array}$ & $\begin{array}{l}\text { Affected by- } \\
\text { alcohol }\end{array}$
\end{tabular}
abuse

\begin{tabular}{lll}
\hline Cortical Gray Matter & + & ++ \\
Frontal lobe & + & ++ \\
Parietal lobe & + & ++ \\
Temporal lobe & $?$ & + \\
White Matter & ++ & ++ \\
Periventricular & ++ & ++ \\
Supraventricular & ++ & ++ \\
Subcortical & $?$ & ++ \\
Subcortical Gray Matter & ++ & ++ \\
Basal ganglia & ++ & ++ \\
Thalamus & + & + \\
Hippocampus & $?$ & + \\
Cerebellum & $?$ & ++ \\
Brainstem (midbrain) & $?$ & + \\
\hline
\end{tabular}

Note: ? = no evidence or unknown; $+=$ some evidence; $++=$ strong evidence

\section{METHODOLOGY}

Dhankut, the group under study are living in small pocket of Dhankuttypura of District Bahraich, Uttar Pradesh and their dialect is Dhankutty in a rhythmic form. They practice cross- cousin and parallel cousin marriage which is a taboo in north India and legally prohibited too under Hindu Marriage Act 1955. Even after having these tribal characteristics, they are not included in the category of scheduled tribe. No literature is available about them either in government records or in district gazetteer. Though they are not agricultural people, cereal selling is their main occupation. Besides, in majority of Dhankut houses, alcohol is prepared from Mahua, as it is grown in plenty in Bahraich district. They are poor and illiterate. With 5\% literacy rate, only three of them are in fourth class government job. Due to poverty, some Dhankut women have also taken to prostitution which also makes this group vulnerable to HIV infection.

Present study was carried out between May-June 2008. It is a primary data based work with the help of participant observation and schedule based interview. Dhankuts are 2687 in number (791 male and 799 female) (as per 2001 census), 1097 (582 boys and 515 girls) are children that is, below the age of 18 years. There- fore, out of 1590 adults Dhankuts, 600 respondents above the age of 18 years have been chosen on the basis of random sampling to analyse the psychoactive effect of alcohol on the Dhankuts. The object of present paper is to see how alcohol impairs various aspects of the immune system and increases the susceptibility to HIV infection amongst this group. The paper also analyses the effects of heavy alcohol use on the severity and progression of HIV infection, including the development of HIV associated CNS disease with the help of various studies done on the subject.

\section{Psychoactive Effects of Alcohol}

Few studies have examined alcohol's direct effects on thoughts and behaviors in relation to sexual risks. One qualitative study conducted with STI clinic patients found that alcohol use to the point of intoxication was believed to lower sexual inhibitions and created barriers in using condoms among both men and women (Simbayi 2004a and Kalichman 2006). This finding is consistent with studies that report that greater quantities of alcohol consumption are associated with engaging in unprotected sex as well as other risk behaviors (Wechsberg et al. 2005). Unfortunately, no research conducted has yet investigated the actual psychoactive effects of alcohol and related mechanisms on subsequent risks for HIV transmission. In summary, people who drink alcohol are at higher risk for HIV than individuals who do not drink. The association between drinking and sexual risks is also observed across a wide array of populations. Any alcohol use at all and drinking greater quantities of alcohol are closely associated with HIV transmission risks.

Present study reflects that 157 (25.67) respondents feel that psychoactive effect of alcohol is that it affects reasoning which leads to unsafe sex and sexual coercion. 404 (67.33) respondents are of the view that use of alcohol makes them insensitive and irresponsible and hence they go for unsafe sex, whereas 42 (7.0) affirm the view that alcohol use causes sexual coercion in intimate relationships (Table 2). The views expressed by respondents are in tune with findings of other studies that alcohol use raises the risks for HIV/AIDS by exposing respondents to unsafe and coercive sex. 
Table 2: Psychoactive effects of alcohol use

\begin{tabular}{|c|c|c|c|c|c|c|c|}
\hline \multirow[t]{2}{*}{$\operatorname{Sex}$} & \multirow[t]{2}{*}{ No. } & \multicolumn{2}{|c|}{ Affects reasoning } & \multicolumn{2}{|c|}{ Unsafe sex } & \multicolumn{2}{|c|}{ Sexual coercion } \\
\hline & & Nos. & $\%$ & Nos. & $\%$ & Nos. & $\%$ \\
\hline Male & 314 & 80 & 25.48 & 208 & 66.24 & 26 & 8.28 \\
\hline Female & 286 & 74 & 25.87 & 196 & 68.53 & 16 & 5.59 \\
\hline Total & 600 & 157 & 25.67 & 404 & 67.33 & 42 & 7.00 \\
\hline
\end{tabular}

\section{Predictors of Alcohol Use}

There are several factors which predict alcohol use in sexual contexts, including community norms, and personal characteristics. Unfortunately, relatively few studies have examined factors that predict alcohol use and sexual risks. Although people who drink often recognize the potential for alcohol to impair their judgment and therefore increase their risks for STI/HIV, this awareness does not necessarily translate to increased perceptions of personal risks for STI and HIV among drinkers (Lewis et al. 2005). Studies suggest that cognitive and personality factors are associated with alcoholrelated sexual risks. Men are significantly more likely to expect that alcohol will increase their sexual desires, whereas women expect the opposite effects of alcohol on sexual desires. In addition, sexual enhancement expectations are related to greater numbers of sex partners and the number of times people regret having had sex (Morojele et al. 2004). The most widely studied personality disposition related to both alcohol use and sexual risk behavior is sensation seeking (Hoyle et al. 2000). Sensation seeking is defined as the propensity to seek optimal sensations through novel and arousing experiencing. The sensation seeking personality disposition reliably predicts engaging in an array of risk behaviors including sexual behaviors and alcohol use across cultures (Zuckerman 1994). Kalichman et al. (2006) reported that sensation seeking predicts both alcohol use in sexual contexts and a cumulative index of HIV transmission risk factors among STI clinic patients. The potential importance of underlying personality characteristics in predicting alcohol use and risk behavior is further supported by research that shows alcohol use is only one of several behaviors that cluster together to increase risk for HIV transmission (Bailey et al. 1999). Drinking alcohol as a means of coping with stress is also related to engaging in higher risk behaviors for HIV transmission (Jones et al. 2005; Wechsberg et al. 2005). Lifestyles that are characterized by alcohol use, especially heavy drinking, can therefore compound HIV risk through multiple channels (Morojele et al. 2004).

\section{Economic Conditions}

Both HIV infection and alcohol use are most concentrated in areas of poverty. Although poverty may well be the foundation for the association between alcohol use and HIV risks, there is surprisingly little research on the connection between poverty, alcohol use, and HIV infection. One factor that connects poverty to alcohol and HIV risk is transactional sex (for example, exchanging sex for money or to meet survival needs). Poverty and unemployment foster both substance use and commercial sex work. In fact, transactional sex is directly related to alcohol use (Dunkle et al. 2004). For example, among women who meet sex partners, nearly half say that their sex partners buy them drinks for sex. The exchange of alcohol or gifts for sex is most common between older men and younger women (Mataure et al. 2002). Women who are involved in sexual exchange are at greatest risk when they work in bars or nightclubs as compared to women who exchange sex in homes (Yadav et al. 2005). The pressures of living in poverty are related to drinking and risks for HIV infection beyond the risks associated with transactional sex. Research conducted in three urban communities in Cape Town, for example, found that sexual risk behaviors were related to perceived stress of poverty (Kalichman et al. 2006). Individuals who perceived greater stress resulting from violence, crime, and discrimination reported greater risks for HIV infection. In this study, alcohol use was related to both perceived stress and HIV risk behavior. Importantly, alcohol use did not account for the association between perceptions of poverty-related social problems and HIV risk behaviors. Perceptions of poverty and alcohol use are therefore related to each other and both are associ- 
ated with HIV risk behaviors. Table 2 shows that 468 (78.0) respondents are of the view that the alcohol is taken to ease the pressures of poverty. 242 (84.62) women are of the view that their husbands take alcohol to avoid the miseries of poverty. The views of respondents are in consonance with the studies done on the point.

\section{Drinking Environments}

Businesses and venues that serve alcohol are often the very places that link alcohol use with risk for HIV infection. Informal alcohol serving establishments, such as private homes where alcoholic beverages are sold and served, are also often the same places where sex partners meet (Morojele et al. 2004). Drinking before sex is more common with non-regular than with regular sex partners (Myer 2002). Drinking establishments may amplify HIV transmission risks by providing a place where high-risk sex encounters can easily unfold (Fritz et al. 2002). Alcohol establishments are often themselves sex venues, where back rooms, back corners, and adjacent buildings or shacks offer locations for sex (Morojele et al. 2006). Places that serve alcohol therefore appear uniquely more linked to HIV transmission risks. Study shows that 82 (13.66) respondents are of the similar view, they take alcohol to drown their sorrows, stress and anxieties and some take it to have the feeling of sensation in their sexual behaviour. The culture of Dhankut is also responsible for their alcohol use. Most of Dhankut families are engaged in the business of making of alcohol at home, which provides them opportunity to indulge in alcoholism by creating drinking environment at their homes itself. 50 (8.33) respondents share this view (Table 3 ).

\section{Alcohol Use, Sexual Risks and Vulnerability to HIV Infection}

Alcohol is the most commonly used psychoactive substance and alcohol is among the most prevalent behaviors associated with sexual risks for HIV and other sexually transmitted infections (STI). Research conducted since the middle 1980s has repeatedly shown that alcohol use is related to sexual risks in several populations, especially among those with the highest rates of HIV infections (Weinhardt and Carey 2001). Alcohol elevates sexual risks through multiple channels, including risk-taking personality characteristics, drinking environments, expectations regarding the effects of alcohol on risk-taking and the psychogenic effects of alcohol on decision making (Cook and Clark 2005).

As many as 50\% of the people where HIV is most prevalent report alcohol use (Shisana et al. 2005). Alcohol use is associated with STI and HIV prevalence. Studies have shown that testing positive for STIs (Gwati et al. 1995; Shaffer et al. 2004) and HIV (Ayisi et al. 2000; Shisana et al. 2005) are independently associated with alcohol use.

Populations that are at greatest risk for HIV/ AIDS also have the greatest history of alcohol use. For example, among drug using commercial sex workers, $26 \%$ report that alcohol was the first drug they ever used, $51 \%$ had started drinking by age 17 , and $18 \%$ drank daily (Wechsberg 2005). Sexually transmitted infection (STI) clinic patients who are also at high risk for HIV commonly report drinking before sex (Simbayi et al. 2004a). Alcohol use is also associated with testing positive for an STI (Feldblum et al. 2000), and daily alcohol use predicts incident TI diagnoses among commercial sex workers (Yadav et al. 2005).

Although frequency of drinking is related to increased sexual risks, the number of times individuals drink appears less important in predicting sexual risks than does the quantity of alcohol consumed. Campbell et al. (2002) and Campbell (2003), for example, found that men and women who drink are significantly more likely to be HIV positive, but frequency of drinking was unrelated to HIV status. Morojele et al. (2004) also found that frequency of alcohol use was not associated with sexual risk behaviors, but quantity of alcohol consumed was related to

Table 3: Predictors of alcohol use

\begin{tabular}{|c|c|c|c|c|c|c|c|}
\hline \multirow[t]{2}{*}{ Sex } & \multirow[t]{2}{*}{ No. } & \multicolumn{2}{|c|}{ Personal } & \multicolumn{2}{|c|}{ Economic } & \multicolumn{2}{|c|}{ Cultural } \\
\hline & & Nos. & $\%$ & Nos. & $\%$ & Nos. & $\%$ \\
\hline $\begin{array}{l}\text { Male } \\
\text { Female }\end{array}$ & $\begin{array}{l}314 \\
286\end{array}$ & $\begin{array}{l}54 \\
28\end{array}$ & $\begin{array}{r}17.20 \\
9.79\end{array}$ & $\begin{array}{l}226 \\
242\end{array}$ & $\begin{array}{l}71.97 \\
84.62\end{array}$ & $\begin{array}{l}34 \\
16\end{array}$ & $\begin{array}{r}10.83 \\
5.59\end{array}$ \\
\hline Total & 600 & 82 & 13.66 & 468 & 78.00 & 50 & 8.33 \\
\hline
\end{tabular}


having greater numbers of recent sex partners. People who drink more heavily and report being intoxicated in sexual situations also report less condom use and more concurrent sex partners, clearly demonstrating higher risk for HIV (Dunkle et al. 2004).

\section{Sexual Coercion}

In traditional societies, men control many aspects of power, including sexual decisionmaking. In the dynamics of power control, sexual and physical violence may be used to reinforce women's subordination and men's claims of sexual privileges. Often, forced sex and wife beating are accepted or even expected expressions of masculinity (Jewkes et al. 2003), while women are usually expected to satisfy their partners' needs. These male behaviors are often more pronounced in the context of substantial age difference between partners, poverty, and low social support (Van der Straten et al. 1995). Males may use violence in response to their partner's accusations of infidelity (Van der Straten et al. 1995) and expose women to unprotected sex (Wingood and Di Clemente 1997) (see, Table 4).

Alcohol consumption, intimate partner physical violence and sexual coercion, although often underreported, are prevalent in many cultures (National Institute on Alcohol Abuse and Alcoholism 1992; Garcia-Moreno and Watts 2000; Maman et al. 2000; Mbulaiteye et al. 2000; Krug et al. 2002; Manfrin-Ledet and Porche 2003). Alcohol consumption before sex is very common (Zablotska et al. 2006). This practice is associated with intimate partner physical violence and sexual coercion. HIV prevalence is significantly higher among women whose partners use alcohol before sex. HIV incidence was also higher among men who reported alcohol use before sex or sexual coercion. Although sexual coercion alone was not statistically associated with higher HIV prevalence, the latter was high among women reporting only sexual coercion. Interestingly, more than half of the women who experienced sexual coercion by their intimate partners reported alcohol use before sex in their relationships. Alcohol may be an important catalyst for intimate partner physical violence and sexual coercion (Cunradi et al. 2002; Weinsheimer et al. 2005). Table 3 shows that the Dhankuts are no exception. 222 (37.0) respondents complained of forced sex, 210 (35.0) of charges of infidelity and 168 (28.0) of unprotected sex. All these factors indirectly create an environment for HIV/ AIDS by forcing partners to go for alternative or multiple partners to satisfy their sexual urge.

Young women in developing countries suffer high rates of HIV infection, and there is concern that male-to-female physical violence and sexual coercion may contribute to their HIV acquisition (Maman et al. 2000; Jewkes et al. 2003; Koenig et al. 2003; Dunkle et al. 2004 Koenig et al. 2004a). Characteristics of the women and their male partners and the nature of the relationship are predictors of violent male behavior (Vlahov et al. 1998). Alcohol use has also been linked to violent behavior (Weinsheimer et al. 2005; Cunradi et al. 2002), and may be a part of the dynamics of sexual control (van der Straten et al. 1995) or may be used as a means to obtain sex against a woman's consent (Rickert et al. 2004). Alcohol use has also been linked to prevalent and incident HIV (Zablotska et al. 2006; Kalichman et al. 2007), and the disinhibiting effect of alcohol consumption is associated with sexual risk behaviors such as inconsistent condom use, increased number of sexual partners, and extramarital partners (Robertson and Plant 1988; Plant 1990; Stall and Leigh 1994; Graves and Leigh 1995; Tveit et al. 1996; Windle 1997; Dolezal et al. 2000). These latter behavioral risk factors have also been linked to HIV acquisition (Hankins 1998; Hearst and Chen 2004; Lau and Muula 2004; Sangani et al. 2004).

Sexual assault and sexual violence is related to alcohol use and HIV transmission risks

Table 4: Types of sexual coercion

\begin{tabular}{|c|c|c|c|c|c|c|c|}
\hline \multirow[t]{2}{*}{$\operatorname{Sex}$} & \multirow[t]{2}{*}{ No. } & \multicolumn{2}{|c|}{ Forced sex } & \multicolumn{2}{|c|}{ Charge of infidelity } & \multicolumn{2}{|c|}{ Unprotected sex } \\
\hline & & Nos. & $\%$ & Nos. & $\%$ & Nos. & $\%$ \\
\hline Male & 314 & 118 & 37.56 & 126 & 40.13 & 70 & 22.29 \\
\hline Female & 286 & 104 & 36.36 & 84 & 29.37 & 98 & 34.27 \\
\hline Total & 600 & 222 & 37.00 & 210 & 35.00 & 168 & 28.00 \\
\hline
\end{tabular}


(Dunkle et al. 2004; Jewkes et al. 2002). Men who have a history of sexual violence are more likely to drink than men who have not been sexually assaultive (Abrahams 2004). Likewise, alcohol use is associated with having been sexually assaulted among women (King et al. 2004). In Uganda, for example, half of women who had been abused reported that their partner drank and one in four reported that their partner drank frequently (Koenig et al. 2003). The association between relationship violence and HIV risk is at least partly accounted for by alcohol use (Phorano et al. 2005). Although it is clear that alcohol consumption and sexual violence are related, their temporal association is less clear. That is alcohol use may precede or follow sexual violence. The power dynamics between men and women are known to foster HIV risk behaviors and alcohol can be used as an instrument for leveraging power in these relationships. Thus, it can be concluded that alcohol impairs various aspects of the immune system leading to unsafe and coercive sex which increases the susceptibility to HIV infection amongst this group.

\section{REFERENCES}

Abrahams N, Jewkes R, Hoffman M, Laubsher, R 2004. Sexual violence against intimate partners in Cape Town: Prevalence and risk factors reported by men. Bulletin of the World Health Organization, 82: 330-337.

Ayisi JG, Van Eijk AM, Ter Kuile FO, Kolczak MS, Otieno JA, Misore AO 2000. Risk factors for HIV infection among asymptomatic pregnant women attending an antenatal clinic in western Kenya. International Journal of STD and AIDS, 11: 393-401.

Bailey RC, Neema S, Othieno R 1999. Sexual behaviors and other HIV risk factors in circumcised and uncircumcised men in Uganda. Journal of Acquired Immune Deficiency Syndromes, 22: 294-301.

Bornstein RA, Fama R, Rosenberger P 1993. Drug and alcohol use and neuropsychological performance in asymptomatic HIV infection. Journal of Neuropsychology 5(3): 254-259.

Campbell C, Williams B, Gilgen D 2002. Is social capital a useful conceptual tool for community level influences on HIV infection? An exploratory case study from South Africa. AIDS Care, 14: 41-54.

Campbell E K 2003. A note on alcohol consumption and sexual behaviour of youths in Botswana. African Sociological Review, 7: 146-161.

Cook RL, Clark D 2005. Is there an association between alcohol consumption and sexually transmitted diseases? A systematic review. Sexually Transmitted Diseases, 32: $156-164$

Cummings JL 1986. Subcortical dementia. Neuropsychology, neuropsychiatry, and pathophysiology. British Journal of Psychiatry, 149: 682-697.
Cunradi CB, Caetano R, Schafer J 2002. Alcohol-related problems, drug use, and male intimate partner violence severity among US couples. Alcoholism: Clinical and Experimental Research, 26: 493-500.

Dingle G A, Oei TPS 1997. Is alcohol a cofactor of HIV and AIDS? Evidence from immunological and behavioral studies. Psychological Bulletin, 122: 56.

Dolezal C, Carballo-Dieguez A, Nieves-Rosa, L, Diaz F 2000. Substance use and sexual risk behavior: Understanding their association among four ethnic groups of Latino men who have sex with men. Journal of Substance Abuse, 11: 323-336.

Dunkle KL, Jewkes RK, Brown HC, Gray GE, McIntryre JA, Harlow SD 2004. Transactional sex among women in Soweto, South Africa: Prevalence, risk factors and association with HIV infection. Social Science and Medicine, 59: 1581-1592.

Dunkle KL, Jewkes RK, Brown HC, Gray GE, Mc Intryre J, Harlow SD 2004. Gender-based violence, relationship power, and risk of HIV infection in women attending antenatal clinics in South Africa. Lancet, 363: 14151421.

Fabris P, Tositti G, Manfrin V 2000. Does alcohol intake affect highly active antiretroviral therapy (HAART) response in HIV-positive patients? Journal of Acquired Immunodeficiency Syndrome, 25: 92-93.

Fein G, Fletcher DJ, Disclafani. V 1998. Effect of chronic alcohol abuse on the CNS morbidity of HIV disease. Alcoholism: Clinical and Experimental Research, 22: 1965-2005.

Feldblum PJ, Kuyoh M, Omari M, Ryan KA, Bwayo JJ, Welsh M 2000. Baseline STD prevalence in a community intervention trial of the female condom in Kenya. Sexually Transmitted Infections, 76: 454-456.

Fritz KE, Woelk GB, Bassett MT, McFarland WC, Routh JA, Tobaiwa O 2002. The association between alcohol use, sexual risk behavior, and HIV infection among men attending beer halls in Harare, Zimbabwe. AIDS and Behavior, 6: 221-228.

Garcia-Moreno C, Watts C 2000. Violence against women: Its importance for HIV/AIDS. AIDS, 14 (Suppl 3): S253-S265.

Graves KL, Leigh BC 1995. The relationship of substance use to sexual activity among young adults in the United States. Family Planning Perspectives, 27: 18-22, 33.

Gwati B, Guli A, Todd CH 1995. Risk factors for sexually transmitted disease amongst men in Harare, Zimbabwe. Central African Journal of Medicine, 41: 179-181.

Hankins C 1998. Changes in patterns of risk. AIDS Care, 10 (Suppl 2): S147-S153.

Hearst N, Chen S 2004. Condom promotion for AIDS prevention in the developing world: Is it working? Studies Family Planning, 35: 39-47.

Hoyle R, Fejfar M, Miller J 2000. Personality and sexual risk taking: A quantitative review. Journal of Personality, 68: 1203-1231.

Jewkes RK, Levin JB, Penn-Kekana LA 2003. Gender inequalities, intimate partner violence and HIV preventive practices: Findings of a South African crosssectional study. Social Science and Medicine, 56: 125134.

Jones DL, Ross D, Weiss SM, Bhat G, Chitalu N. 2005. Influence of partner participation on sexual risk behavior reduction among HIV-positive Zambian women. Journal of Urban Health, 82: iv92-iv100.

Kalichman SC, Simbayi LC, Kagee A, Toefy Y, Cain D, Cherry C 2006. Association of poverty, substance use, 
and HIV transmission risk behaviors in three South African communities. Social Science and Medicine, 62: 1641-1649.

Kalichman SC, Simbayi LC, Kaufman M, Cain D, Jooste S 2007. Alcohol use and sexual risks for HIV/AIDS in sub- Saharan Africa: Systematic review of empirical findings. Prevention Science, 8: 141-151.

Kaslow RA, Blackwelder WC, Ostrow DG1989. No evidence for a role of alcohol or other psychoactive drugs in accelerating immunodeficiency in HIV-1-positive individuals. Journal of the American Medical Association, 261: 3424-3429.

King G, Flisher AJ, Noubary F, Reece R, Marais A, Lombard C 2004. Substance abuse and behavioral correlates of sexual assault among South African adolescents. Child Abuse and Neglect, 28: 683-696.

Koenig MA, Lutalo T, Zhao F, Nalugoda F, WabwireMangen F, Kiwanuka N 2003. Domestic violence in rural Uganda: Evidence from a community-based study. Bulletin of the World Health Organization, 81: 5360.

Krug EG, Dahlberg LL, Mercy JA, Zwi AB, Lozano R 2002. World Report on Violence and Health. Geneva: World Health Organization.

Lau C, Muula AS 2004. HIV/AIDS in Sub-Saharan Africa. Croatian Medical Journal, 45: 402-414.

Lewis JJC, Garnett GP, Mhlanga S, Nyamukapa CA, Donnelly CA, Gregson S 2005. Beer halls as a focus for HIV prevention activities in rural Zimbabwe. Sexually Transmitted Diseases, 32: 364-369.

Maman S, Campbell J, Sweat MD, Gielen AC 2000. The intersections of HIV. The Journal of the Association of Nurses in AIDS Care and violence: Directions for future research and interventions. Social Science and Medicine, 50: 459-478.

Manfrin-Ledet L, Porche DJ 2003. The state of science: Violence and HIV infection in women. Journal of Association of Nurses in AIDS Care, 14: 56-68.

Masliah E, Ge N, Mucke L 1996. Pathogenesis of HIV-1 associated neurodegeneration. Critical Reviews in Neurobiology, 10(1): 57-67.

Mbulaiteye SM, Ruberantwari A, Nakiyingi JS, Carpenter LM, Kamali A, Whitworth JA 2000. Alcohol and HIV: A study among sexually active adults in rural southwest Uganda. International Journal of Epidemiology, 29: 911-915.

Morojele NK, Kachienga MA, Mokoko E, Nkoko MA, Parry CDH, Nkowane AM 2006. Alcohol use and sexual behaviour among risky drinkers and bar and shebeen patrons in Gauteng province, South Africa. Social Science and Medicine, 62: 217-227.

Morojele NK, Kachienga MA, Nkoko MA, Moshia AM, Mokoko E, Parry CDH 2004. Perceived effects of alcohol use on sexual encounters among adults in South Africa. African Journal of Drug and Alcohol Studies, 3: $1-20$.

Myer L, Matthews C, Little F 2002. Condom use and sexual behaviors among individuals procuring free male condoms in South Africa: A prospective study. Sexually Transmitted Diseases, 29: 239-241.

National Institute on Alcohol Abuse and Alcoholism 1992. Alcohol Alert. Rep. No. 15:PH 311

Phorano OD, Nthomang K, Ntseane D 2005. Alcohol abuse, gender-based violence and HIV/AIDS in Botswana: Establishing the link based on empirical evidence. Sahara: Journal of Social Aspects of HIV/AIDS, 2: 188-202.
Penkower L, Dew MA, Kingsley L 1995. Alcohol consumption as a cofactor in the progression of HIV infection and AIDS. Alcohol, 12: 547-552.

Petry N 1999. Alcohol use in HIV patients: What we don't know may hurt us. International Journal of STD and AIDS, 10:561-570.

Plant MA 1990. Alcohol, sex and AIDS. Alcohol, 25: 293301

Pol S, Artru P, Thepot V, Berhelot P, Nalpas B 1996. Improvement of the CD4 cell count after alcohol withdrawal in HIV-positive alcoholic patients. AIDS, 10: $1293-1294$

Price RW, Brew B, Sidtis J, Rosenblum M, Scheck AC, Cleary P 1988. The brain in AIDS: Central nervous system HIV-1 infection and AIDS dementia complex. Science, 239: 586-592.

Rickert VI, Wiemann C, Vaughan RD, White JW 2004. Rates and risk factors for sexual violence among the ethnically diverse sample of adolescents. Archives of Pediatrics and Adolescent Medicine, 158: 1132-1139.

Robertson JA, Plant MA 1988. Alcohol, sex and risks of HIV infection. Drug and Alcohol Dependence, 22: 75-78.

Rosenbloom MJ, Pfefferbaum A, Sullivan E 1995. Structural brain alterations associated with alcoholism. Alcohol Health and Research World, 19(4):266- 272.

Sangani P, Rutherford G, Wilkinson D 2004. Population based interventions for reducing sexually transmitted infections, including HIV infection. Cochrane Database of Systematic Reviews, CD 001220.

Shisana O, Rehle T, Simbayi L, Parker W, Bhana A, Zuma K 2005. South African National HIV Prevalence, Incidence, Behaviour and Communication Survey. Cape Town: Human Sciences Research Council Press.

Simbayi LC, Kalichman SC, Jooste S, Mathiti V, Cain D, Cherry C 2004a. Alcohol use and sexual risks for HIV infection among men and women receiving sexually transmitted infection clinic services in Cape Town, South Africa. Journal of Alcohol Studies, 65: 434-442.

Stall R, Leigh BC 1994. Understanding the relationship between drug or alcohol use and high risk sexual activity for HIV transmission: Where do we go from here? Addiction, 89: 131-134.

Sullivan E 2000. Human brain vulnerability to alcoholism: Evidence from neuroimaging studies. In: Review of NIAAA's Neuroscience and Behavioral Research Portfolio. National Institute on Alcohol Abuse and Alcoholism Research Monograph.Bethesda, MD, the Institute, 34: 473-508.

Szabo G 1999. Consequences of alcohol consumption on host defense. Alcohol and Alcoholism, 34(6): 830-841.

Tozzi V, Balestra P, Galgani S 1999. Positive and sustained effects of highly active antiretroviral therapy on HIV-1 associated neurocognitive impairment. AIDS, 13: 18891897

Tveit KS, Nyfors A, Nilsen A 1996. Casual sex, extramarital sex, condom use and alcohol intake among heterosexual patients attending an STD clinic in Norway. Acta Dermato-Venereologica, 76: 150-153.

Tyor WR, Middaugh LD 1999. Do alcohol and cocaine abuse alter the course of HIV-associated dementiacomplex? Journal of Leukocyte Biology, 65: 475.

Van der Straten A, King R, Grinstead O, Serufilira A, Allen S 1995. Couple communication, sexual coercion and HIV risk reduction in Kigali, Rwanda. AIDS, 9: 935-944.

Vlahov D, Wientge D, Moore J, Flynn C, Schuman P, Schoenbaum EE 1998. Violence among women with or at risk for HIV infection., AIDS and Behavior, 2: $53-60$. 
Wang Y, Watson RR 1995. Is alcohol consumption a cofactor in the development of acquired immunodeficiency syndrome? Alcohol, 12:105-109.

Wechsberg WM 2005.The Pretoria women's health co-op study. South African Community Epidemiology Network on Drug Use (SACENDU): Monitoring Alcohol and Drug Trends. Tygerberg, South Africa: Medical Research Council.

Wechsberg WM, Luseno WK, Lam WK 2005. Violence against substance-abusing South African sex workers: Intersection with culture and HIV risk. AIDS Care, 17: S55-S64.

Weinhardt L, Carey MP 2001. Does alcohol lead to sexual risk behavior? Annual Review of Sex Research, 12: $125-157$.

Weinsheimer RL, Schermer CR, Malcoe LH, Balduf LM, Bloomfield LA 2005. Severe intimate partner violence and alcohol use among female trauma patients. The Journal of Trauma, 58: 22-29.
Windle M 1997. The trading of sex for money or drugs, sexually transmitted diseases (STDs), and HIV-related risk behaviors among multi-substance using alcoholic inpatients. Drug and Alcohol Dependence, 49: 33-38.

Wingood GM, DiClemente RJ 1997. The effects of an abusive primary partner on the condom use and sexual negotiation practices of African-American women. American Journal of Public Health, 87: 1016-1018.

Yadav G, Saskin R, Ngugi E, Kimani J, Keli F, Fonck K 2005. Associations of sexual risk taking among Kenyan female sex workers after enrollment in an HIV-1 prevention trial. JAIDS, 38: 329-334.

Zablotska IB, Gray RH, Serwadda D, Nalugoda F, Kigozi G Sewankambo N 2006. Alcohol use before sex and HIV acquisition: a longitudinal study in Rakai, Uganda. AIDS, 20: 1191-1196.

Zuckerman M 1994. Behavioral Expressions and Biological Bases of Sensation Seeking. New York: Oxford University Press. 